\title{
Historical glass mosaic tesserae: a multi-analytical approach for their characterization
}

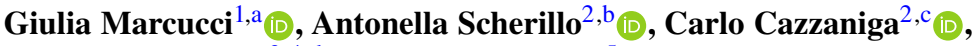 \\ Quentin Lemasson $^{3,4, d_{(\mathbb{D}}}$, Roberto Lorenzi $^{5, \mathrm{e}_{\mathbb{C}}}$, Massimiliano Clemenza $^{1, \mathrm{f}} \mathrm{f}_{\mathbb{D}}$, \\ Maria Pia Riccardi ${ }^{6}$, Daniela Di Martino ${ }^{1, g_{(D}}$ \\ ${ }^{1}$ Dipartimento di Fisica "G. Occhialini”, Università Milano-Bicocca, and INFN, Piazza della Scienza 3, \\ 20126 Milan, Italy \\ 2 ISIS, Neutron and Muon Source, Rutherford Appleton Laboratory, Chilton-Didcot OX11 0QX, UK \\ ${ }^{3}$ Centre de Recherche Et de Restauration Des Musées de France, 14 quai François Mitterrand, 75001 Paris, \\ France \\ ${ }^{4}$ FR3506 NewAGLAE, Ministère de La Culture/Chimie ParisTech, 14 quai François Mitterrand, 75001 Paris, \\ France \\ 5 Dipartimento di Scienza dei Materiali, Università Milano-Bicocca, via Cozzi 55, 20125 Milan, Italy \\ ${ }^{6}$ Dipartimento di Scienze della Terra e dell'Ambiente and Arvedi Laboratorio-sede di Pavia, Università degli \\ Studi di Pavia, Via Ferrata 9, 27100 Pavia, Italy
}

Received: 14 April 2021 / Accepted: 23 June 2021

(c) The Author(s) 2021

\begin{abstract}
We present a completely non-destructive approach to analyse a set of historical glass mosaic tesserae. Aim of the study is to obtain a qualitative and quantitative characterization of the glass matrix in terms of elements and mineralogical phases. Several nondestructive techniques have been applied like Particle-Induced X-ray Emission, ParticleInduced Gamma-ray Emission, micro-Raman spectroscopy, neutron resonance capture analysis and neutron activation analysis in order to combine different technique strengths and to explore the effect of different range sizes for the analysis. Yet, best practices require the use of combined analysis and different skills. The paradigm of a non-destructive multi-analytical approach is suggested for a comprehensive investigation in non-homogeneous real samples, like historical glass mosaic tesserae.
\end{abstract}

\section{Introduction}

Glass production in antiquity has followed different recipes in different chronological periods, depending on the availability of raw materials and on glass manufacturing techniques. As a matter of fact, glass was a widespread material from late Egypt to modern times, and many

\footnotetext{
a e-mail: giulia.marcucci@unimib.it

b e-mail: antonella.scherillo@stfc.ac.uk

c e-mail: carlo.cazzaniga@stfc.ac.uk

d e-mail: quentin.lemasson@culture.gouv.fr

e e-mail: roberto.lorenzi@unimib.it

f e-mail: massimiliano.clemenza@unimib.it

g e-mail: daniela.dimartino@unimib.it (corresponding author)
} 
archaeological studies discriminate provenance, manufacturing techniques and chronological issues of glass specimens on the basis of different elemental and mineralogical compositions [1]. In general, a mosaic glass is a silica-based material with many fining, colouring and opacifying agents. Just to give an example of the complexity of glass composition, mediaeval and post-mediaeval glass was categorized in four types based on its alkali concentration: (a) Na-rich glass, with $\mathrm{Na}_{2} \mathrm{O}>12 \mathrm{wt} \%$ and $\mathrm{K}_{2} \mathrm{O}<5$ wt \%; (b) K-rich glass, with $\mathrm{K}_{2} \mathrm{O}$ between 10 and $15 \mathrm{wt} \%$ and $\mathrm{Na}_{2} \mathrm{O}<5 \mathrm{wt} \%$; (c) mixed alkali glass, with intermediate relatively high values for both sodium and potassium; and (d) high-lime low-alkali HLLA glass, where $\mathrm{Na}_{2} \mathrm{O}+$ $\mathrm{K}_{2} \mathrm{O}<10 \mathrm{wt} \%$ [2]. Similar categories exist for colourants and opacifying agents (like calcium antimonate, lead antimonate and cassiterite or their allotropes to cite a few). Thus, the search for specific elements, but also mineralogical phases (for the colouring and opacifying agents), is very important, and the use of completely non-destructive techniques is highly desirable. In the literature, the study of archaeological glass composition is based on consolidated techniques, like inductively coupled plasma-optical emission spectrometry (ICP-OES) [3] and electron microprobe analysis (EMPA) [4-6]. Yet, ICP-OES requires sampling, which is not acceptable for many archaeological artefacts, and EMPA is often invasive. Moreover, due to the limited areas investigated, the results obtained by these techniques not always return a comprehensive description of inhomogeneous samples. Other techniques could be applied, and we tried to explore different types of analysis in different size ranges (from microns to centimetres spatial ranges of examination). In fact, mosaic glasses are archaeological glasses whose beauty is connected to their many hues, colours and transparencies, which derive from the composition and location of different mineralogical phases and chromophore ions modifying the starting glass structure. Therefore, the samples cannot be considered homogeneous, and depending on the areas examined, different results could be obtained due to the different spatial distribution of modifying ions and phases.

We carried out an in-depth study by different techniques, in order to obtain the composition of a set of historical mosaic tesserae. Ion beam analyses (IBA), like Particle-Induced X-ray Emission (PIXE) and Particle-Induced Gamma-ray Emission (PIGE), micro-Raman spectroscopy and neutron-based experiments were performed to check the presence of specific elements and mineralogical phases. However, we stress that not all analyses can describe the bulk of the specimens. IBA can hardly penetrate the surface layers, does not discriminate among allotropes and has a limited region of acquisition (spot measurements). Micro-Raman spectroscopy can inspect the inner parts of the specimens only when transparent samples are analysed. Moreover, the investigated area that a single measurement can reach is micrometric

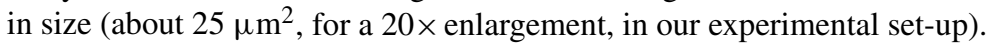

We thus propose, in order to have a non-destructive bulk characterization, a new multianalysis route studying a set of historical mosaic tesserae by a wide number of surface techniques. Finally, the combination with neutron-based techniques, like neutron resonance capture analysis (NRCA) and neutron activation analysis (NAA), will allow for a comprehensive elemental bulk characterization in a non-destructive way.

The combined use of surface (like IBA and Raman) and bulk (NRCA and NAA) techniques will provide the elemental and mineralogical phases composition. The results suggest the use of a multidisciplinary approach to have a comprehensive quantitative description of mosaic glasses, evidencing the most appropriate non-destructive technique for the study of different glass characteristics. 


\section{Materials and methods}

In this study, a set of 18 glass tesserae have been chosen as specimens to be tested for a multi-analytical study as part of an ongoing methodological research [7-10]. The mosaic tesserae (described and displayed in Table 1) have different dating and provenance, and most of them are coloured opaque tesserae. The sample set is composed by four Greek tesserae dating back to I-II century AD and one to the XI century AD, three Syrian tesserae dating back to the VIII century AD, and ten Italian tesserae coming from Basilica di San Vitale in Ravenna dating back to the VI century AD. The samples do not present any visible patina or alteration layer on their surface. The protocol is a combination of non-destructive analytical techniques described below, including PIXE/PIGE, micro-Raman spectroscopy and neutronbased analysis. As it will be detailed, PIXE/PIGE measurements cannot be described as bulk ones. In general, micro-Raman spectroscopy, for non-transparent samples, allows only single-spot and surface analysis. In order to have a quantitative bulk analysis of the samples, neutron-based techniques have been applied, exploiting the neutrons penetration depth of the order of several centimetres, even in dense material. This property relies on the different nature of radiation-matter interaction that distinguishes neutrons with respect to charged particles. Therefore, neutrons allow both surface and bulk properties to be measured in a non-destructive manner.

\subsection{Particle-Induced X-ray Emission and Particle-Induced Gamma-ray Emission}

Ion beam PIXE/PIGE analyses were performed at AGLAE facility (Centre de Recherche et Restauration des Musées de France-C2RMF), based in the Palais du Louvre in Paris (France). A $3 \mathrm{MeV}$ proton beam, extracted through a $0.1 \mu \mathrm{m}$ thick $\mathrm{Si}_{3} \mathrm{~N}_{4}$ window (surface $1 \mathrm{~mm}^{2}$ ), hits the samples with a beam spot about $50 \mu \mathrm{m}$ wide. For PIXE analysis, four $50 \mathrm{~mm}^{2}$ silicon drift detectors (SDDs) were used with a $50 \mu \mathrm{m}$ aluminium filter to enhance the detection of high-energy X-rays, while one SDD with a smaller solid angle was used in a helium atmosphere to enhance the response to low-energy X-rays. In this configuration, it is possible to identify the elements with $Z>11$ and to measure their concentrations with a lower limit of detection for higher atomic number and depending on irradiation conditions. An ultrapure germanium detector was used to detect in parallel the $\gamma$-rays from $60 \mathrm{keV}$ to $2 \mathrm{MeV}$ for PIGE analysis, fundamental for the measurement of sodium concentration. In order to reduce the effects of sampling inhomogeneities, each measurement was taken on a wider area $\left(500 \times 500 \mu \mathrm{m}^{2}\right)$. Moreover, each sample was measured in two or more different points. This was necessary since the surface could display heterogeneities at a micrometric scale. Though we used a larger analysis spot, with respect to the micrometric beam spot, reaching a sub-millimetre area, other problems can affect the measurements, like non-planar surface or the presence of inclusions.

PIXE data analysis was performed following the procedures described in [11]. PIGE normalization is needed to reach an inner part of the samples. In fact, though the estimated range of proton penetration in the analysed material is of the order of about a hundred $\mu \mathrm{m}$, the effective depth of analysis depends on the element. For sodium, the X-ray information comes from the first microns, whereas for $\gamma$-rays, being of much higher energy, the information comes from a bigger volume upper than $30 \mu \mathrm{m}$ [12]. Precise quantitative measurements of element concentrations were taken and controlled by regularly using a set of calibration references, in particular standard glasses like BRILL A, B, D. Errors can be estimated as in [13], with values ranging from 1 to $10 \%$, depending on the concentrations. 
Table 1 Glass tesserae samples listed by codes, pictures, weight, provenance, dating and colour

\begin{tabular}{|c|c|c|c|c|}
\hline Sample code & Sample image & Weight (mg) & Provenance and dating & Colour \\
\hline DEL1 & & 24.98 & $\begin{array}{l}\text { Delos } \\
\text { Monastery-Greece, } \\
\text { 1st-2nd AD }\end{array}$ & Opaque blue \\
\hline DEL2 & & 29.25 & $\begin{array}{l}\text { Delos } \\
\text { Monastery-Greece, } \\
\text { 1st-2nd AD }\end{array}$ & Opaque red \\
\hline DEL3 & & 6.91 & $\begin{array}{l}\text { Delos } \\
\text { Monastery-Greece, } \\
\text { 1st-2nd AD }\end{array}$ & Opaque green \\
\hline DEL4 & & 5.71 & $\begin{array}{l}\text { Delos } \\
\text { Monastery-Greece, } \\
\text { 1st-2nd AD }\end{array}$ & Transparent blue \\
\hline DAFNI & & 3.56 & $\begin{array}{l}\text { Dafni } \\
\text { Monastery-Greece, } \\
\text { 11th AD }\end{array}$ & $\begin{array}{l}\text { Opaque red with a golden } \\
\text { layer }\end{array}$ \\
\hline SYG1 & & 1.17 & Syria, 8th AD & $\begin{array}{l}\text { Transparent with golden } \\
\text { inclusions }\end{array}$ \\
\hline SYG2 & & 14.70 & Syria, 8th AD & Opaque dark green \\
\hline SYG3 & & 34.36 & Syria, 8th AD & Opaque green \\
\hline SVC12 & & 2.85 & Ravenna-Italy, 6th AD & Opaque red \\
\hline SVP27 & & 8.48 & Ravenna-Italy, 6th AD & Opaque green \\
\hline SVP46 & & 1.63 & Ravenna-Italy, 6th AD & Opaque green \\
\hline SVP59 & & 2.83 & Ravenna-Italy, 6th AD & Opaque black \\
\hline SVP58 & & 1.44 & Ravenna-Italy, 6th AD & Opaque black \\
\hline
\end{tabular}


Table 1 continued

\begin{tabular}{|c|c|c|c|c|}
\hline Sample code & Sample image & Weight (mg) & Provenance and dating & Colour \\
\hline SVP57 & & 4.95 & Ravenna-Italy, 6th AD & Opaque black \\
\hline SVR24 & & 4.01 & Ravenna-Italy, 6th AD & Opaque pink \\
\hline SVR25 & & 22.50 & Ravenna-Italy, 6th AD & Opaque green \\
\hline SVR26 & & 1.80 & Ravenna-Italy, 6th AD & Opaque red \\
\hline SVR27 & & 7.25 & Ravenna-Italy, 6th AD & Opaque red/striped \\
\hline
\end{tabular}

Sample sizes can be derived by sample images, superimposed on a square background (side of $0.5 \mathrm{~cm}$ )

\subsection{Raman spectroscopy}

A micro-Raman spectroscopy study has been applied, which allowed the identification of mineralogical phases used as opacifiers dispersed in the glass matrix as well as the characterization of some of the glass matrices themselves. The Raman spectrometer used to analyse the glass tesserae is a LabRam HORIBA Jobin Yvon. The spectrometer works in backscattered configuration and is coupled to an optical microscope to selectively analyse components of heterogeneous samples on a micrometre scale. Three objectives are available to change the dimension of the sample area under investigation: $20 \times, 50 \times$ and $100 \times$, which corresponds to spot sizes of about $10 \mu \mathrm{m}, 5 \mu \mathrm{m}$ and $2 \mu \mathrm{m}$ in diameter, respectively. The sample holder consists of a motorized XY stage, characterized by a spatial resolution of $0.1 \mu \mathrm{m}$. The spectral resolution of the Raman spectrometer is about $2 \mathrm{~cm}^{-1}$. Two different lasers have been used: $\mathrm{He}-\mathrm{Ne}$ at $633 \mathrm{~nm}$ and $\mathrm{Ar}^{+}$at $488 \mathrm{~nm}$, whose choice was related to the possible presence of photoluminescence signals.

The spectral position of the detection system was calibrated with a standard of crystalline silicon before any measurement.

The attribution of the Raman signatures of the crystalline inclusions has been made by comparison with data in the literature as well as in the RRUFF database [14], while the assignment of the glass matrix by comparison with the literature only.

\subsection{Neutron-based techniques}

Neutron resonance capture analysis (NRCA) and neutron activation analysis (NAA) have been conducted at the ISIS Neutron and Muon Source (UK). NRCA and NAA return isotopic and elemental composition of the samples in major, minor and trace elements. NRCA measurements were taken at the INES (Italian Neutron Experimental Station) beamline of ISIS, which is characterized by a white pulsed neutron beam moderated by a water moderator at $295 \mathrm{~K}$, and a sample-to-moderator distance $L_{0} \approx 22.8 \mathrm{~m}$ [15]. Being ISIS a pulsed neutron 
source, time-of-flight (ToF) spectroscopy measurements, such as NRCA, are possible. This means that the energy of the absorbed neutrons is directly related to their time of flight, which is the actual physical quantity measured.

The presence of resonance structures in neutron-induced reaction cross sections is the basis of the NRCA technique. For each nuclide, resonances appear at specific energies; thus, these sharp peaks are fingerprints of nuclides and can be used to determine the elemental and isotopic composition of materials [16].

All mosaic tesserae were singularly packed in aluminium foils without pre-treatment and then irradiated with the INES neutron flux. During irradiation, neutron capture reactions occur in the sample and prompt $\gamma$ radiation is emitted. The $\gamma$-rays' arrival time on the detectors is recorded using three yttrium aluminium perovskite (YAP) crystals coupled with photomultipliers. The arrival time of the $\gamma$ cascade following the neutron capture reaction corresponds to the time of flight (and therefore energy) of the neutron captured by the sample.

Each NRCA spectrum has been acquired alternating measurements with and without the sample to correct the data from background contributions. The NRCA data were processed using the Mantid software [17].

NAA measurements were taken by transferring each sample at the ChipIR beamline after irradiation on INES and by measuring the induced radioactivity with a high-purity germanium (HPGe) detector [18]. The HPGe is housed in a lead shielding to limit the background signal. Each irradiated tessera has been placed at contact, at the centre of the HPGe active surface. In most cases, especially when several isotopes with different half-lives were produced, the measurement of the radioactivity of the samples has been repeated a few times. The first measurements after irradiation have been needed to detect the $\gamma$-rays of isotopes with relatively short half-life, while measurements after the decay of short-lived isotopes have allowed higher sensitivity to isotopes with lower activity and longer decay time. For each sample, three measurements of a few minutes have been taken, followed by several measurements of one hour. However, it required about $10 \mathrm{~min}$ to transfer samples from the INES beamline to the HPGe detector. Isotopes with short half-lives cannot readily be detected. A prior energy calibration of the detector has been performed using radioactive samples of ${ }^{137} \mathrm{Cs},{ }^{241} \mathrm{Am}$, ${ }^{60} \mathrm{Co}$ and ${ }^{40} \mathrm{~K}$.

We highlight that neutron-based techniques were seldom used, though being a nondestructive technique very useful in heritage science studies. Some interesting applications were shown in [16], though NRCA was never applied to mosaic samples.

\section{Results and discussion}

\subsection{PIXE and PIGE}

Table 2 reports PIXE results, after PIGE normalization, obtained by the procedure described in Sect. 2.1. Elemental composition is first obtained by PIXE, and the oxide concentration can be derived by PIGE. We can note that all elements (starting from sodium oxide) have been detected, when above the detection limit.

Most of the samples display the same sodium oxide concentration, within $10 \%$ uncertainty. This can be an indicator of the same glass matrix, while other oxide content can differ too.

On the contrary, four samples (SVP57/SVR25/DEL4/DAFNI) show different concentrations for sodium at least in 2 of the 4 points analysed. While one sample is clearly a multilayer sample (DAFNI), other samples did not show higher heterogeneities typical of a multilayer structure. Careful attention should be paid when composition data are discussed for man- 


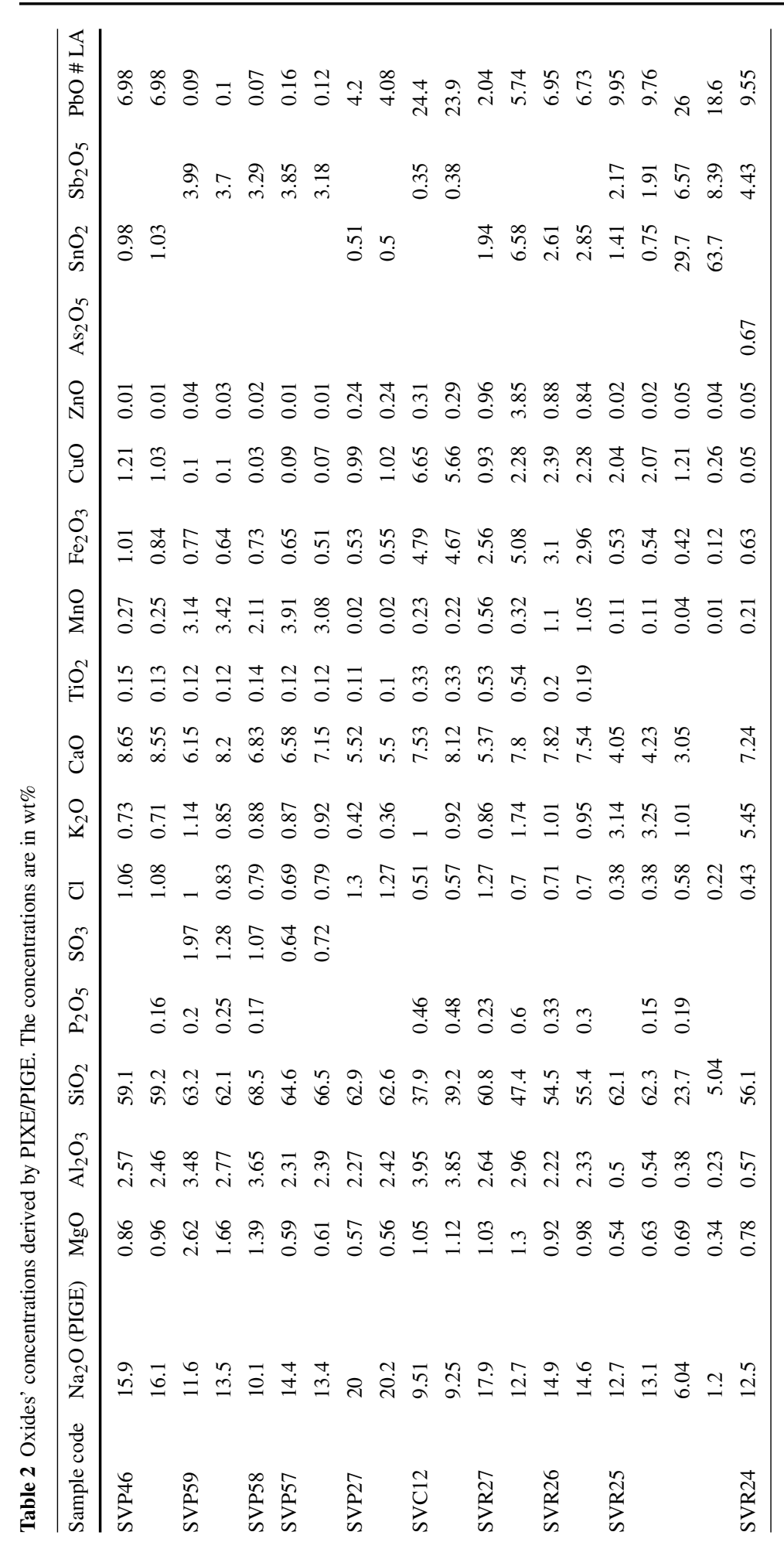




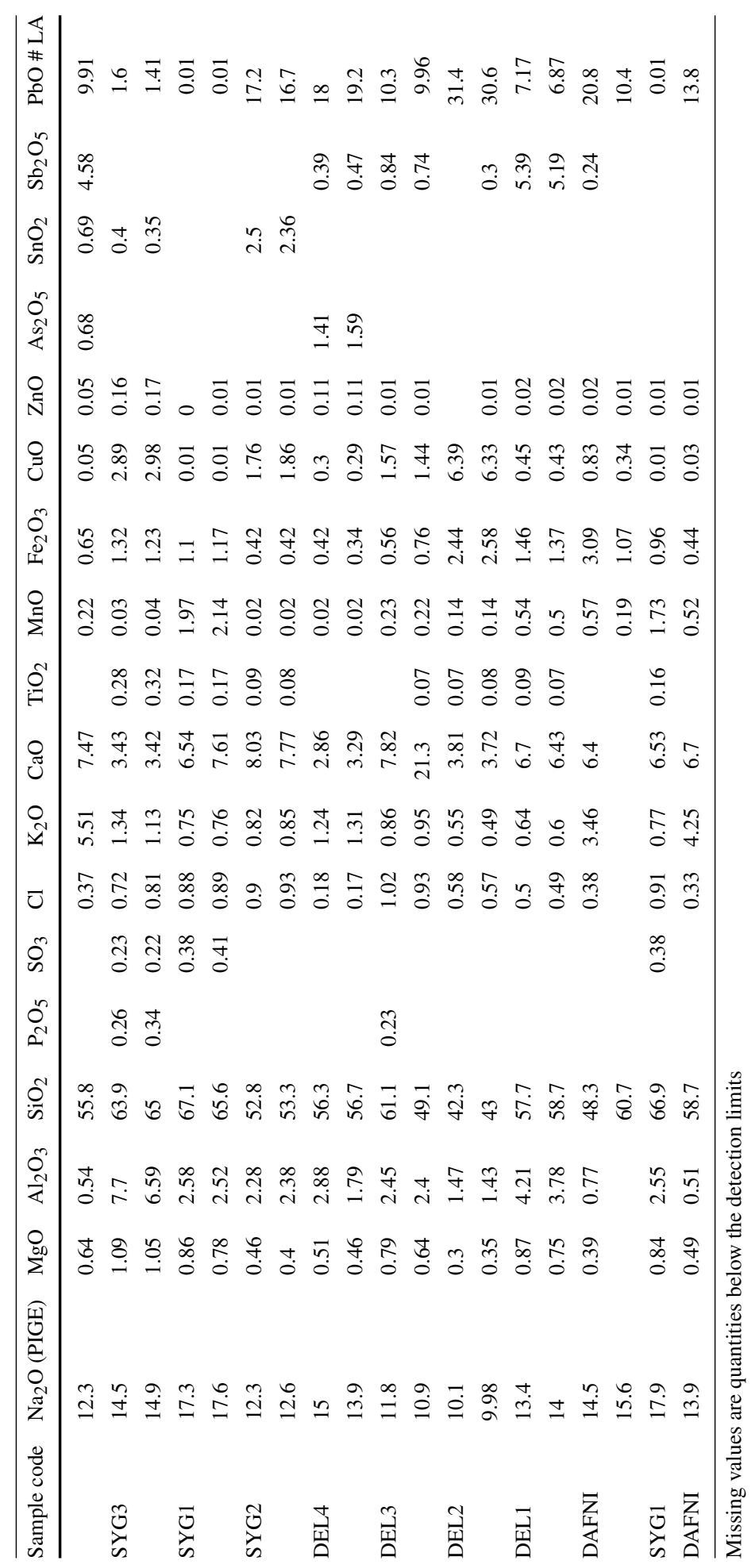


ufacturing and provenance attributions. For example, SVR25 has one measured point with a very low silica content and a very high Sn-oxide content, maybe due to the presence of some Sn-based crystalline phase. On the other hand, for SVP57 similar contents of silica are measured in the two spots analysed, while different contents of S-, Mn-, Fe- and K-oxides are obtained, maybe due to a different glass matrix.

\subsection{Raman spectroscopy}

Raman scattering can provide different information on glass tesserae depending on the investigated area and its magnification. Raman spectra of glass mosaic tesserae generally exhibit broad bands due to the vibration of the amorphous glass network. Instead, the presence of narrow Raman peaks is due to crystalline phases acting as opacifiers.

The Raman spectrum of the amorphous matrix of the mosaic tessera DAFNI is an example of the Raman signature of the network of our glasses (see supplementary materials S1). Two main regions can be distinguished: one between 300 and $600 \mathrm{~cm}^{-1}$ associated with the stretching and bending vibration of the glassy structure, and a region between 900 and $1200 \mathrm{~cm}^{-1}$ associated with the stretching vibration of species containing non-bridging oxygens. The basic unit of silicates (glassy or crystalline) is the $\mathrm{SiO}_{4}$ tetrahedron. Pure amorphous silica can be described as a $3 \mathrm{D}$ network of $\mathrm{SiO}_{4}$ tetrahedra sharing their oxygen atoms and forming $\mathrm{Si}-\mathrm{O}-\mathrm{Si}$ bridges [19]. However, glass mosaic tesserae were produced by using different raw materials and intentionally adding glass modifiers mainly to reduce the melting point. Impurities replace the $\mathrm{Si}^{4+}$ covalent bounded atoms decreasing the $\mathrm{Si}-\mathrm{O}$ bridges and the connectivity of the glassy network and creating different tetrahedra species, known as $\mathrm{Q}^{n}$ configuration, where $n$ denotes the number of bridging oxygens. The small band around $800 \mathrm{~cm}^{-1}$ is assigned to the motion of Si against its tetrahedral oxygen cage [20]. Previous Raman spectroscopy studies of the $\mathrm{Si}-\mathrm{O}$ stretching and bending component of the glassy network have shown the possibility to differentiate between compositional families e.g. lead-based and alkali-based glasses [21-23]

Following the literature [21-23], an attempt of spectral deconvolution has been carried out with the aim of separating the different vibrational species [24] (see supplementary materials $\mathrm{S} 1$ and S2). The relative intensity of the $\mathrm{Q}^{n}$ components (calculated as the ratio between the Gaussian area and the total area of the stretching region) and their corresponding wavenumbers are shown in Fig. 1. Samples DEL3, DEL4 and SYG3 show similar characteristics, while DAFNI $Q^{n}$ components suggest a different composition from the others as DAFNI $Q^{3}$ intensity is the lowest, while its $Q^{1}$ and $Q^{2}$ intensities are the highest. This result implies two possible conclusions: the DAFNI mosaic tessera has more modifiers than the Sirian and Delos tesserae, or its modifiers are based on heavy elements, such as lead, which break the network bounds more invasively.

A further consideration can be made on the reproducibility of the Raman measurements. Depending on the investigated region of the glasses, the same samples show different Raman spectra and wavenumber positions of the $Q^{n}$ components due to the heterogeneous structure of the glass tesserae. Thus, Raman spectroscopy analysis should be conducted on several sections of the tesserae to obtain a more comprehensive description about the structure.

The polymerization index [25] has been derived for samples DAFNI and SYG3, and the results are in accordance with PIXE/PIGE results. In fact, the polymerization index of DAFNI ranges between $0.46-0.48$, and according to [19], it reflects a high-lead concentration in the glass and a low melting temperature, while the SYG3 sample shows higher values of the polymerization index (0.54-0.59), and therefore, the two types of mosaic tessera belong to different compositional families. 


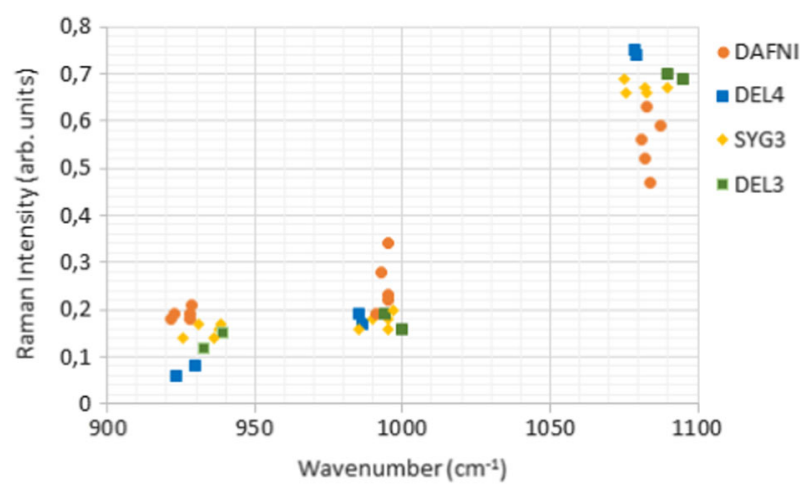

Fig. 1 Area of the different $Q^{n}$ species as a function of their corresponding wavenumber (centre of the Gaussian peak). See supplementary materials for details
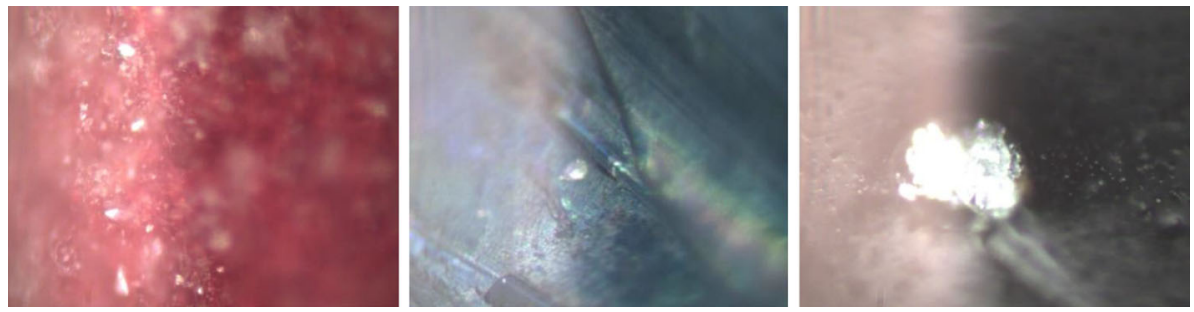

Fig. 2 From the left: view of sample DEL2, DEL4 and SVP57 at the microscope camera. The crystalline phases detected in these regions are cuprite, diopside and thenardite, respectively. The image dimensions are $0.37 \times 0.55 \mathrm{~mm}^{2}$

By selecting with the microscope camera a particular area of the sample where a defined structure attributable to a crystalline phase was morphologically visible (Fig. 2), it was often possible to associate mineralogical phases to the Raman spectra of these structures. In fact, Raman spectra (see Fig. 3) gave evidence of the presence of crystal structures that could often be associated with phases both related to the production history of the artefact and to its use. These structures are quite similar to those of mineralogical phases, of which the Raman spectra are well known.

In our set of samples, we detected several phases listed in Table 3. The Raman signatures of calcium-antimonates have quite recently been recognized in the scientific literature on mosaic tesserae [26]. In this case, the assignment of the Ca-antimonate phase has been made through reference spectra found in the literature. Concerning sample SVR25, the Sn-based crystalline phase suggested by PIXE/PIGE results could be assigned to cassiterite. All acquired spectra of sample SYG2 were characterized by strong luminescence; thus, it does not present any useful Raman signal for phases identification.

\subsection{Neutron resonance capture analysis}

NRCA relies on the analysis of resonance capture spectra obtained by detecting prompt $\gamma$-rays emitted after a neutron capture reaction in the sample. A qualitative analysis of the NRCA spectra has been conducted by indexing the observed resonances and comparing the centre of each measured peak to tabulated libraries [27]. Only four mosaic tesserae show 

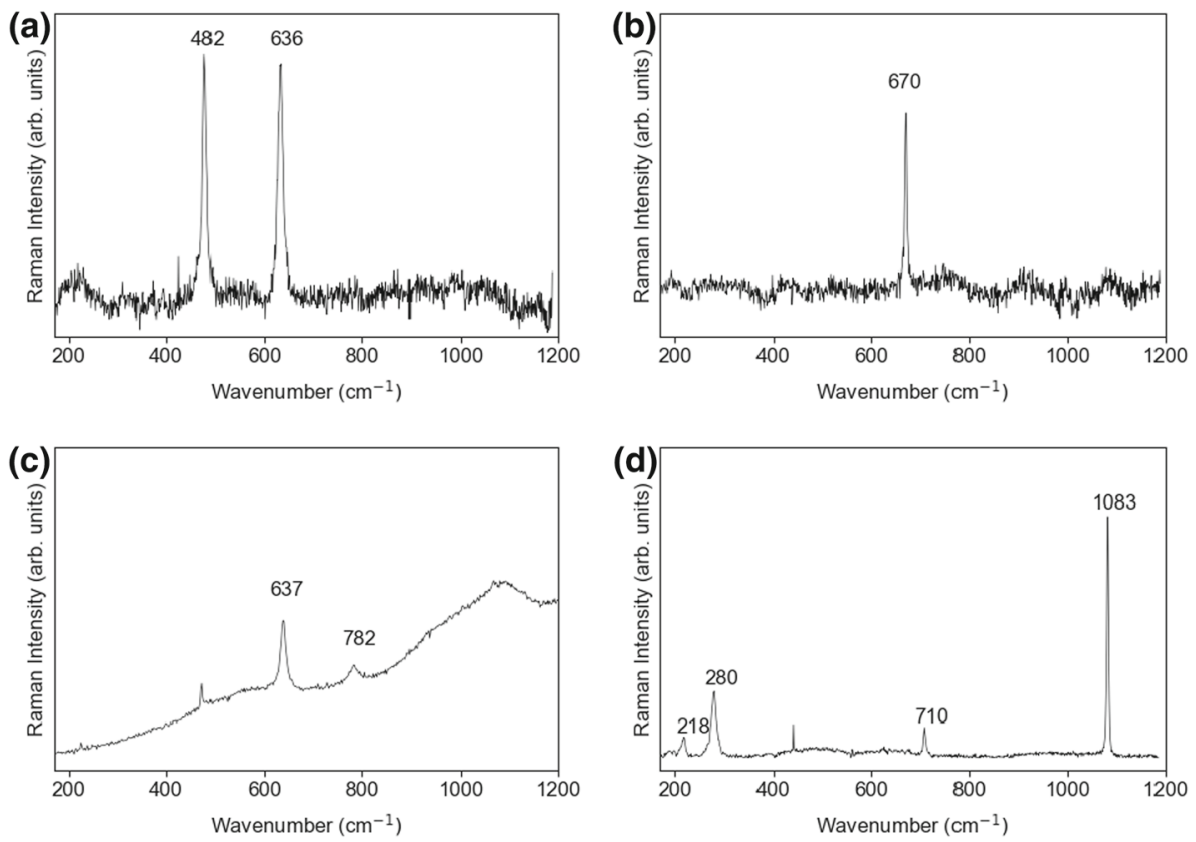

Fig. 3 a Representative Raman spectrum of the crystalline phase $\mathrm{Ca}_{2} \mathrm{Sb}_{2} \mathrm{O}_{7}$ identified within DEL1. b Representative Raman spectrum of the crystalline phase $\mathrm{CaSbO}_{6}$ identified within SVP57. c Representative Raman spectrum of the crystalline phase $\mathrm{SnO}_{2}$ identified within SYG3. d Representative Raman spectrum of the crystalline phases $\mathrm{Sn}_{3} \mathrm{O}_{2}(\mathrm{OH})_{2}$ and $\mathrm{CaCO}_{3}$ identified within DEL2. The spectra were acquired using the $632.8 \mathrm{~nm}$ HeNe laser line with $5 \mathrm{~mW}$ of power at the source. Other Raman spectra, according to Table 3, are available as supplementary materials (S3)

NRCA spectra with resonance peaks (Figs. 4, 5). Samples SVR25, SVR24 and DEL1 show peaks at $302 \mu \mathrm{s}, 356 \mu \mathrm{s}, 418 \mu \mathrm{s}$ and $660 \mu \mathrm{s}$ that can be assigned to antimony.

The presence of copper in sample DEL2 was derived assigning the two peaks at 36.5 and $68.5 \mu \mathrm{s}$, which correspond to the most intense resonances of the copper cross section for neutron capture reactions. One peak related to lead is also visible at low time of flight $(30 \mu \mathrm{s})$ in the NRCA spectrum of sample DEL2.

This result obtained by NRCA is consistent with the opaque red colour of the sample DEL2: from the literature [28, 29] it is known that the combination of these two elements was employed by the craftsmen to obtain the red tint, given by copper oxides and copper ions, whose precipitation is facilitated by the presence of lead (see [28], pages 105-128).

In the other cases, we can conclude that NRCA signal of expected elements is not visible due to their amount lower than the corresponding detection limit, which is not constant among the elements, but it depends on the neutron capture cross section of the single element.

Moreover, the INES set-up is not yet optimized to perform quantitative NRCA, and thus, only qualitative information can be obtained through this technique at present. Therefore, the NRCA technique is suitable for detecting antimony and thus for deducing the presence of Sb-based opacifiers on a larger investigation area compared to Raman spectroscopy and ion beam analysis. The same consideration can be made for copper, which is related to colouring functions in the glass. Another advantage of NRCA is the capability of detecting the presence of lead as opposed to neutron activation analysis (as will be explained in Sect. 3.4). 
Table 3 Raman phase assignments of inclusions

\begin{tabular}{llll}
\hline Sample & Peak position $\left(\mathrm{cm}^{-1}\right)$ & Phase assignment & \\
\hline DEL1 & 482,636 & $\mathrm{Ca}_{2} \mathrm{Sb}_{2} \mathrm{O}_{7}$ & Ca-antimonate \\
DEL2 & 218 & $\mathrm{Sn}_{3} \mathrm{O}_{2}(\mathrm{OH})_{2}$ & Hydroromarchite R090060 \\
& $280,710,1083$ & $\mathrm{CaCO}_{3} \mathrm{Cu}_{2} \mathrm{O}$ & Calcite R040070 \\
& 200,220 & & Cuprite R050374 \\
DEL3 & $280,710,1083$ & $\mathrm{CaCO}_{3}$ & Calcite R040070 \\
DEL4 & $320,390,664,1013$ & $\mathrm{MgCaSi}_{2} \mathrm{O}_{6}$ & Diopside R040009 \\
SYG3 & 637,782 & $\mathrm{SnO}_{2}$ & Cassiterite R060563 \\
SVP27 & 281,712 & $\mathrm{CaMn}_{2}+\left(\mathrm{CO}_{3}\right)_{2}$ & Kutnohorite R060473 \\
SVP46 & $286,717,1090$ & $\left.\mathrm{CaMg}_{2} \mathrm{CO}_{3}\right)_{2}$ & Dolomite R050129 \\
SVP57 & 670 & $\mathrm{CaSbO}_{6}$ & Ca-antimonate \\
& $450,466,621,633,650,893$, & $\mathrm{Na}_{2} \mathrm{SO}_{4}$ & Thenardite R040183 \\
& $1101,1133,1153$ & & Ca-antimonate \\
SVR24 & $203,337,481,510,636$ & $\mathrm{Ca}_{2} \mathrm{Sb}_{2} \mathrm{O}_{7}$ & Lead antimonate \\
& 334,507 & $\mathrm{~Pb}_{2} \mathrm{Sb}_{2} \mathrm{O}_{7}$ & Cassiterite R060563 \\
SVR25 & $476,540,634,777$ & $\mathrm{SnO}_{2}$ & Lead antimonate \\
& $340,456,506$ & $\mathrm{~Pb}_{2} \mathrm{Sb}_{2} \mathrm{O}_{7}$ & .
\end{tabular}

Where a numerical code beginning with " $\mathrm{R}$ " is given, reference spectra are taken from the RRUFF database [14]

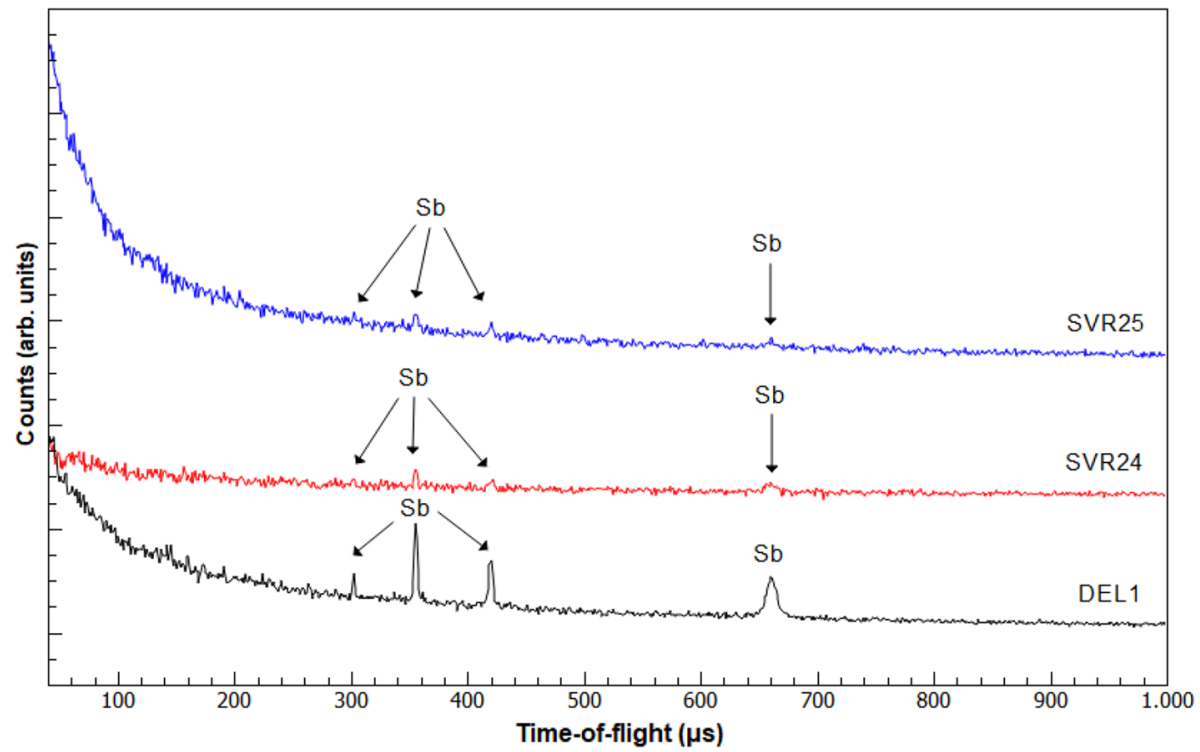

Fig. 4 Neutron resonance capture analysis spectra with elements assignment of mosaic samples SVR25, SVR24 and DEL1. Four peaks related to antimony are visible in each spectrum

\subsection{Neutron Activation Analysis}

For each tessera, elemental composition obtained by NAA is presented in Table 4. 


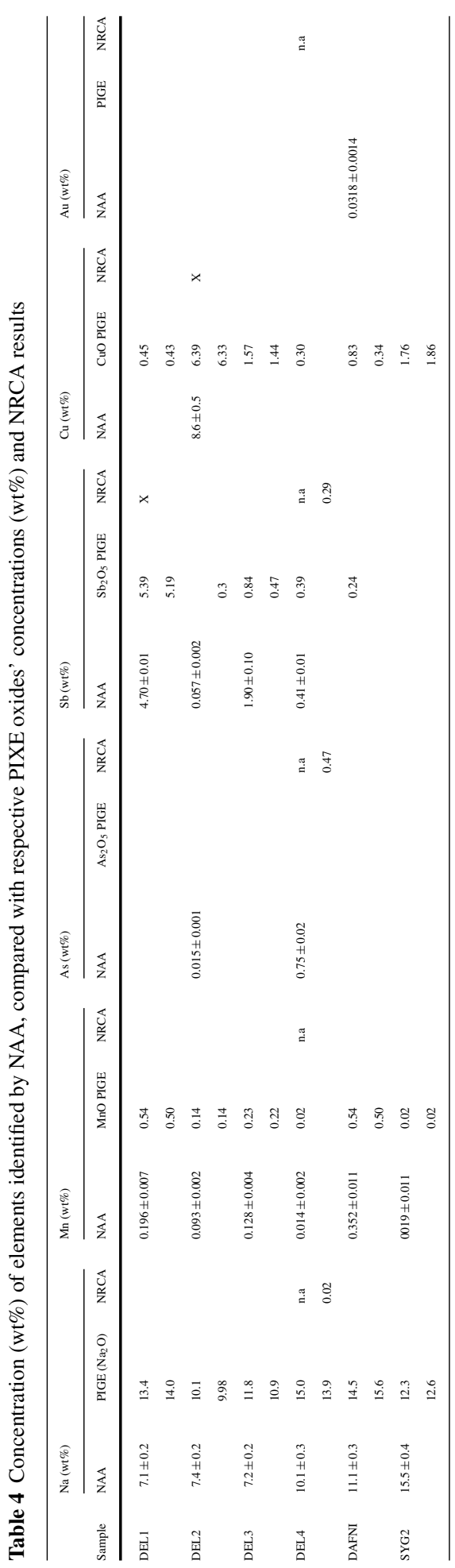




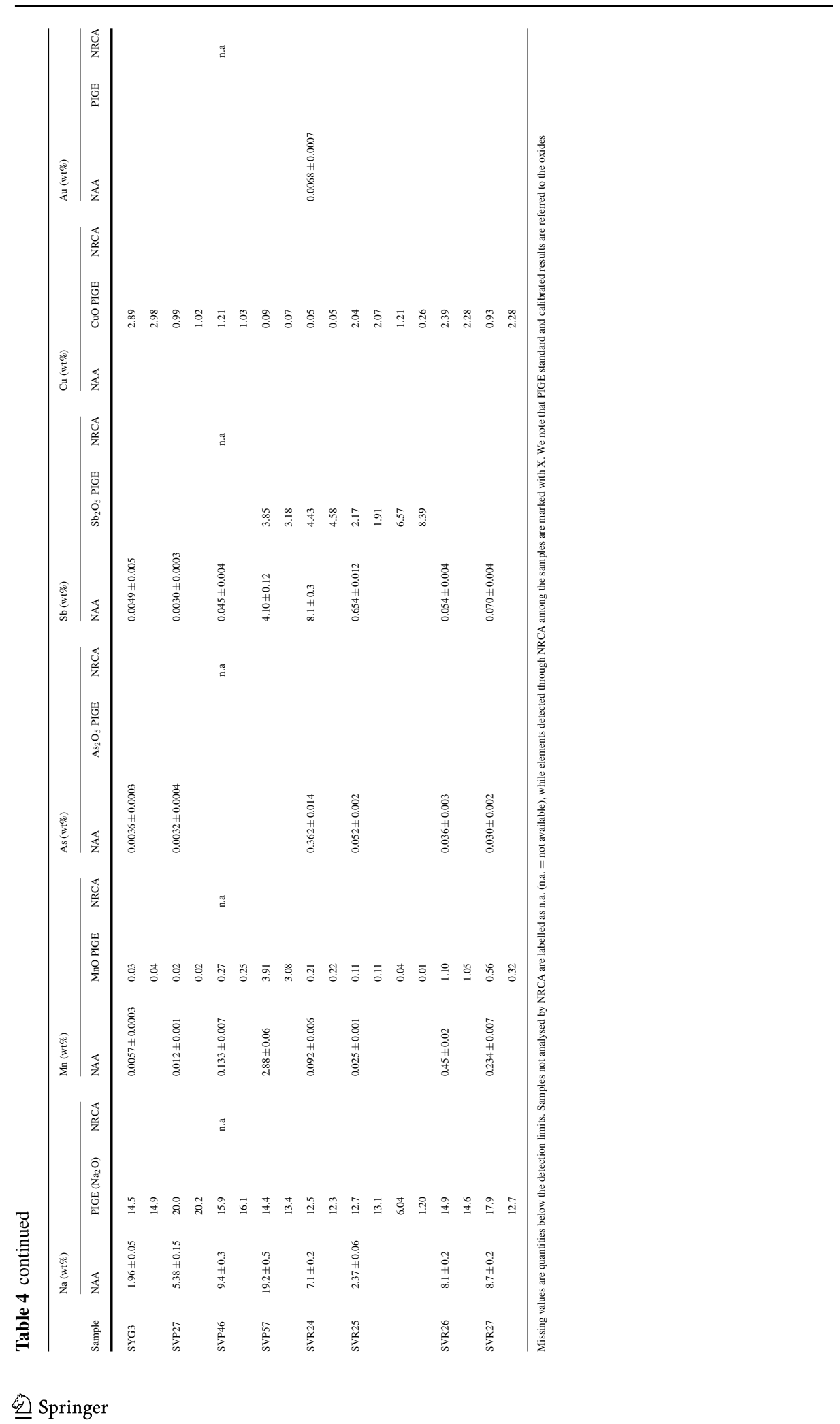




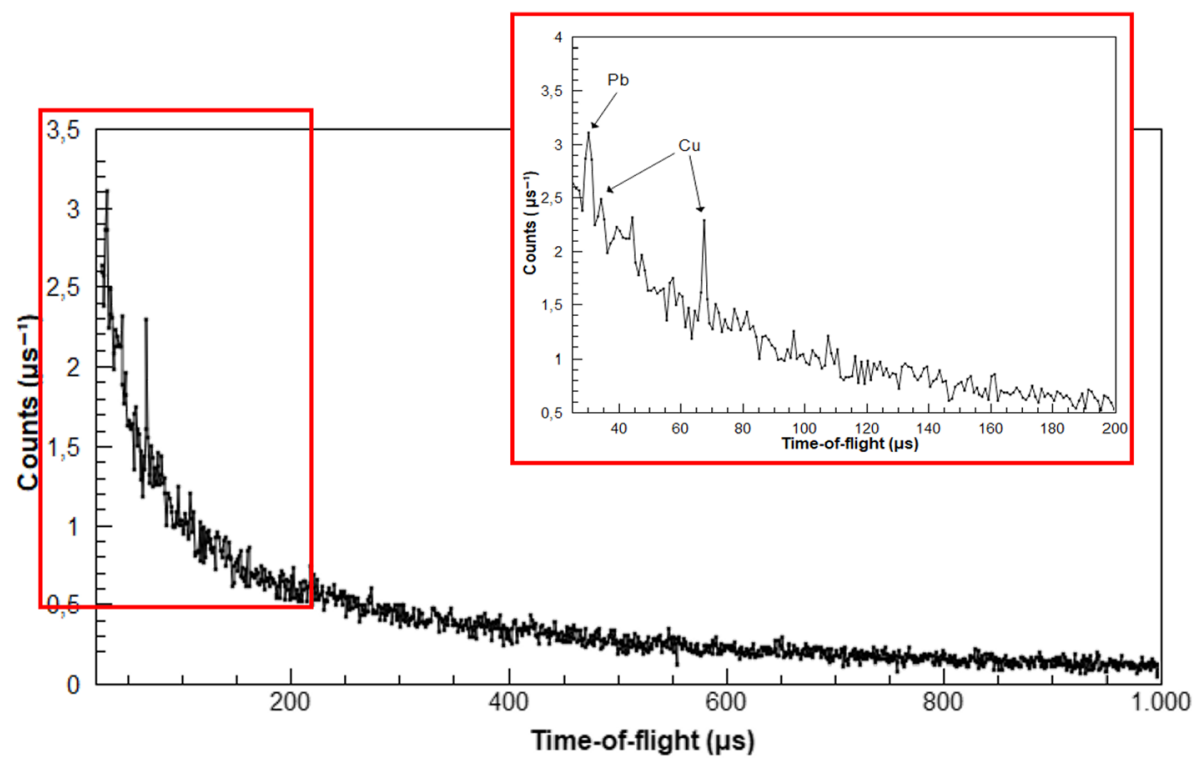

Fig. 5 Neutron resonance capture analysis spectra with elements assignment of mosaic sample DEL2. Two peaks related to copper are visible (enlarged in the inset)

We can note that not all the elements can be measured by NAA in our set-up. The detection limit of the neutron activation technique is not easy to derive as it is different for each element. In fact, several element characteristics affect its detection limit. First, the neutron capture cross section: elements with very high neutron capture cross sections can be detected in part per million, such as gold or arsenic (Fig. 6). Other elements are quite difficult to detect due to very low probability of interaction with neutrons, such as lead. The half-life of the radioactive isotopes is also crucial. Very fast decay times (compared to the waiting time between sample irradiation and activity measurements) lead to loss of the possibility of detecting such isotopes. As an example, the radioisotope $\mathrm{Mg}-27$ produced by thermal neutron activation has a half-life of about ten minutes that is comparable with our waiting time. Thus, we cannot detect the presence of magnesium in our samples, although it is a typical element constituting glass mosaic tesserae.

Other important parameters are the $\gamma$ energy compared to the background and interferences due to sample activation.

In Table 4, NAA results are compared with the corresponding ones obtained by PIGE, and there is a good agreement (within errors) for many samples. We can also observe that the quantitative analysis provided by NAA is representative of the bulk composition, while PIXE values are often lower or higher, depending on the morphology of the investigated area. Regarding antimony and arsenic, NAA is more sensitive with respect to PIXE while we have the opposite situation for copper. Moreover, through NAA we could quantify the Au content, while to get the same information through PIXE, a priori calibration with a metallic reference standard is needed. 


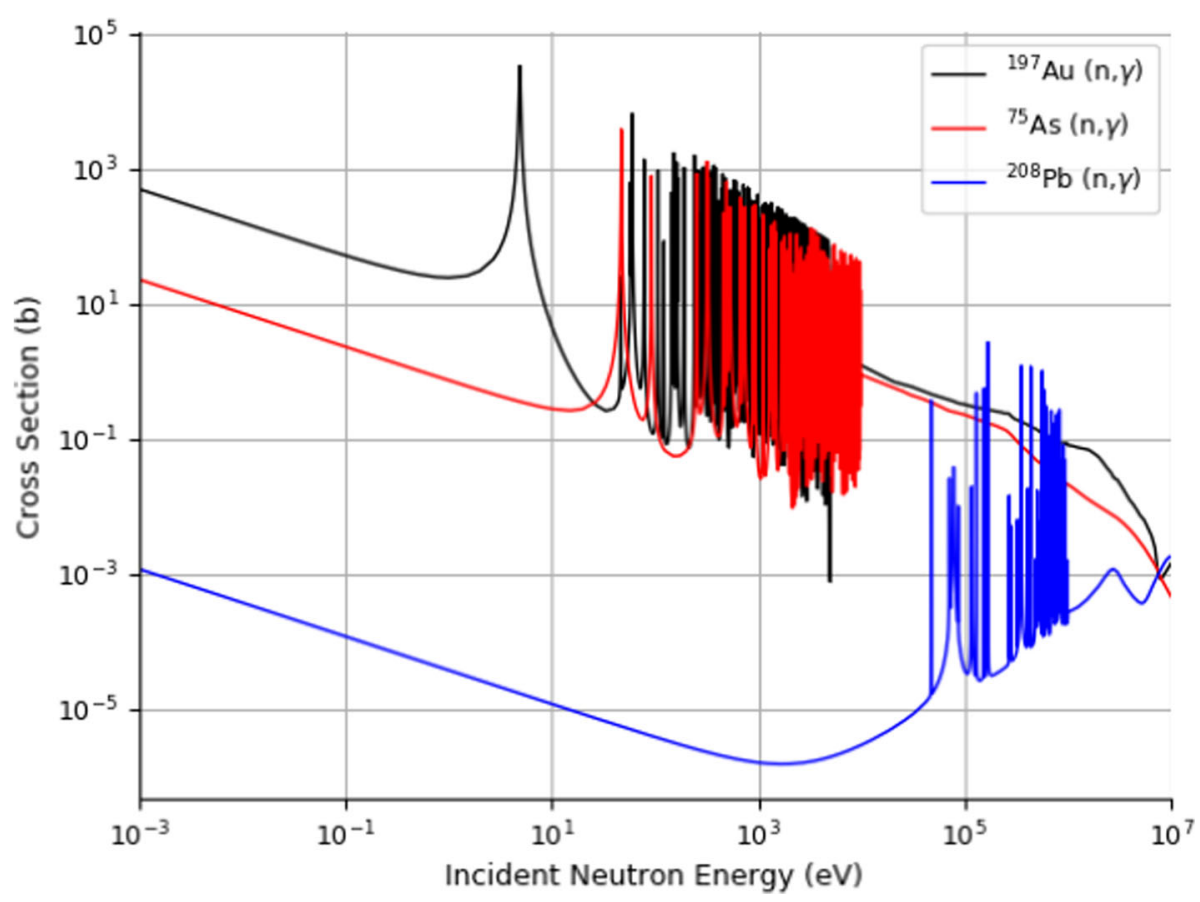

Fig. 6 Neutron capture cross section of As-75, Au-197 and Pb-208 [27]

\section{Conclusions}

Results obtained by a multi-analytical approach have evidenced that there is not a technique of choice if the aim is to study mosaic tesserae by a completely non-destructive analysis. In fact, all the considered techniques have advantages and limitations, as explained below. A summary of the major features of these analytical methods is reported in Table 5.

Ion beam PIXE/PIGE analyses return compositional information in terms of a wide range of oxides and elements. With PIGE analysis, low-Z elements, such as sodium, can also be detected. On the other hand, this technique does not provide bulk information as the investigated area is limited to tens of micrometres in thickness. It is worth mentioning that PIXE/PIGE results cannot always reflect the mean composition of the tesserae. In fact, being a surface and spot analysis, it can be affected by an alteration layer or the composition can be altered by the presence of a micro-crystal cluster in the investigated section.

Raman spectroscopy is a well-known technique for micrometric analysis of heterogeneous compounds. Its major advantages include crystal specificity and non-destructiveness, the first very appreciated for univocal phases identification and the latter mandatory for studies on archaeological and precious artefacts. A related characteristic to consider is the high spatial and spectral resolution. Moreover, spectra measured on the glass matrix and within inclusions were in agreement with PIXE/PIGE analysis as far as lead glasses analysis and microcrystalline phases detection. For our studies, another remarkable advantage is the possibility to conduct analysis by benchtop instrumentation. However, Raman spectroscopy is a single-spot technique and limited to qualitative information regarding opacifiers and glass matrix composition. Databases of minerals Raman spectra are very useful for phase identification, and the availability of a large number of compositional studies of glasses and mosaic tesserae in the literature allows to have a good statistic for differentiating between glass fam- 


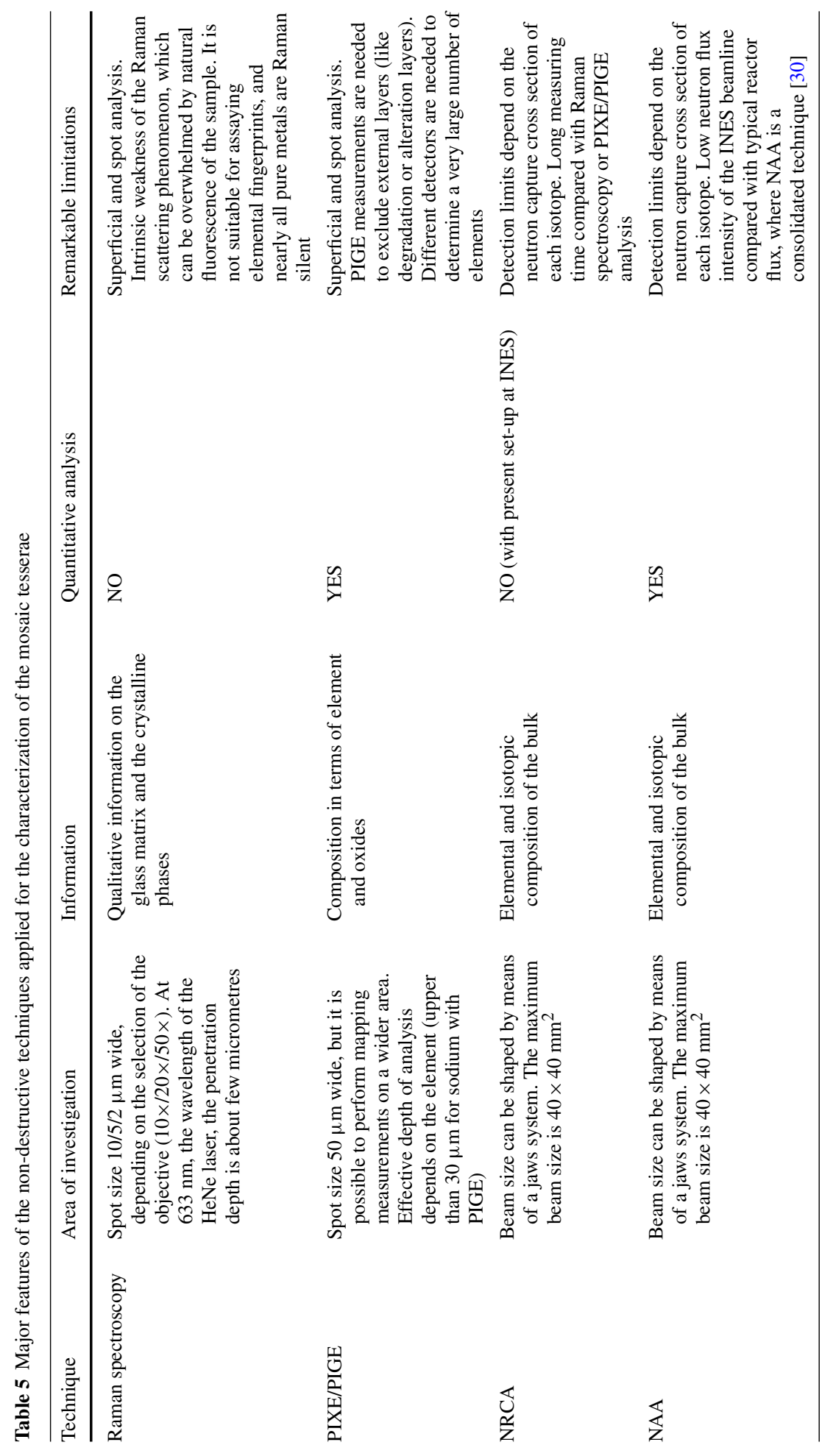


ilies. By examining different areas of the same glass tessera with Raman spectroscopy, the heterogeneity of this kind of sample is highlighted, as well as by the PIXE and PIGE results.

On the contrary, neutron-based techniques provide compositional information of the whole bulk of the mosaic tesserae in terms of elements, overcoming effects of potential alteration layer and the heterogeneity of historical glasses. In fact, neutrons are a high penetrating probe and the small size of our samples compared to the dimension of the INES neutron beam allows them to be irradiated in the whole volume.

Thus, NRCA and NAA can be considered non-destructive analyses complementary to the previous superficial ones. However, they are mainly limited by the neutron reactions' cross sections of the elements present within the glasses, and consequently, not all elements can be detected with our experimental conditions (neutron energy, irradiation time and measure time).

Therefore, in the archaeometric search for the best characterization route to be used for the glass mosaic tesserae composition determination, we can conclude that when limited to the use of non-destructive techniques it is necessary to consider a multi-analytical approach. Only exploring different methodologies, different spatial resolutions and inner parts can be reached and an in-depth characterization of the tesserae can be obtained.

Supplementary Information The online version contains supplementary material available at https://doi. org/10.1140/epjp/s13360-021-01696-2.

Acknowledgements This work was partially supported within the CNR-STFC Agreement 2014-2020, concerning collaboration in scientific research at the spallation neutron source ISIS (UK). Financial support by the Access to Research Infrastructures activity in the Horizon 2020 Programme of the EU (IPERION CH Grant Agreement n. 654028) is gratefully acknowledged (FIXLAB: AGLAE, France).

Authors' contributions D.D. and G.M. conceived the idea of a new protocol for mosaic glass samples. Q.L, M.P.R., G.M. and D.D. performed PIXE/PIGE experiments, G.M. carried out Raman measurements in collaboration with R.L., G.M., C.C. and A.S. performed the neutron experiments. All authors gave contributions in the data analysis and results discussion. D.D. and G. M. wrote the manuscript in consultation with all authors.

Funding Open access funding provided by Università degli Studi di Milano - Bicocca within the CRUI-CARE Agreement. Partial fundings have been received as explained in the acknowledgement section.

Data Availability Statement This manuscript has associated data in a data repository. [Authors' comment: The raw/processed data required to reproduce these findings are available from the corresponding author on reasonable request.]

Code availability Not applicable.

\section{Declarations}

Conflict of interest The authors declare no conflicts or competing interests.

Open Access This article is licensed under a Creative Commons Attribution 4.0 International License, which permits use, sharing, adaptation, distribution and reproduction in any medium or format, as long as you give appropriate credit to the original author(s) and the source, provide a link to the Creative Commons licence, and indicate if changes were made. The images or other third party material in this article are included in the article's Creative Commons licence, unless indicated otherwise in a credit line to the material. If material is not included in the article's Creative Commons licence and your intended use is not permitted by statutory 
regulation or exceeds the permitted use, you will need to obtain permission directly from the copyright holder. To view a copy of this licence, visit http://creativecommons.org/licenses/by/4.0/.

\section{References}

1. J. Henderson, Ancient Glass: An Interdisciplinary Exploration (Cambridge University Press, New York, 2013)

2. D. Dungworth, T. Cromwell, D. Ashurst, C. Cumberpatch, D. Higgins, H. Willmott, Post Mediev. Archaeol. 40(1), 160-190 (2006)

3. R. Arletti, S. Quartieri, G. Vezzalini, Period. Mineral. 75, 25-38 (2006)

4. R. Arletti, C. Fiori, M. Vandini, Archaeometry 52(5), 796815 (2010)

5. A. Silvestri, S. Tonietto, G. Molin, P. Guerriero, J. Archaeol. Sci. 39(7), 2177-2190 (2012)

6. A. Silvestri, F. Nestola, L. Peruzzo, Microchem. J. 124, 811-818 (2016)

7. D. Di Martino, A. Galli, M. Martini, J. Raman Spectrosc. 43, 18241827 (2012)

8. C.B. Azzoni, D. Di Martino, V. Marchesi, B. Messiga, M.P. Riccardi, Archaeometry 47, 381388 (2005)

9. C.B. Azzoni, D. Di Martino, C. Chiavari, M. Martini, E. Sibilia, M. Vandini, Archaeometry 44, 543-554 (2002)

10. C.B. Azzoni, D. Di Martino, B. Messiga, M.P. Riccardi, Period. Mineral. Vol. Spec. Archaeom. Cult. Herit. 71, 73-85 (2002)

11. L. Pichon, T. Calligaro, Q. Lemasson, B. Moignard, C. Pacheco, Nucl. Instrum. Methods Phys. Res. Sect. B Beam Interact. Mater. Atoms 363, 48-54 (2015). https://doi.org/10.1016/j.nimb.2015.08.086

12. I. Biron, S. Beauchoux, Meas. Sci. Technol. 14(9), 15641578 (2003). https://doi.org/10.1088/0957-023 3/14/9/308

13. M. Radepont, Q. Lemasson, L. Pichon, B. Moignard, C. Pacheco, Measurement 114, 501-507 (2018). https://doi.org/10.1016/j.measurement.2016.07.005

14. B. Lafuente, R.T. Downs, H. Yang, N. Stone, The power of databases: the RRUFF project, in Highlights in Mineralogical Crystallography. ed. by T. Armbruster, R.M. Danisi (W De Gruyter, Berlin, 2015), pp. $1-30$

15. A. Pietropaolo, G. Festa, F. Grazzi, E. Barzagli, A. Scherillo, E.M. Schooneveld, F. Civita, EPL 95, 48007 (2011). https://doi.org/10.1209/0295-5075/95/48007

16. N. Kardjilov, G. Festa, Neutron Methods for Archaeology and Cultural Heritage (Springer, Berlin, 2017)

17. O. Arnold et al., Nucl. Instrum. Methods Phys. Res. Sect. A 764, 156-166 (2014)

18. C. Cazzaniga, A. Scherillo, A. Fedrigo, D. Raspino, F. Grazzi, C.D. Frost, Neutron activation analysis of archaeological artefacts using the ISIS pulsed neutron source. AIP Advances (2021). https://doi.org/10. $1063 / 5.0043935$

19. P. Colomban, A. Tournie, L. Bellot-Gurlet, J. Raman Spectrosc. 37, 841-852 (2006)

20. F.L. Galeener, A.E. Greissberger, Phys. Rev. B 27(10), 6199-6204 (1983)

21. P. Colomban, F. Treppoz, J. Raman Spectrosc. 32, 93102 (2001)

22. P. Colomban, G. Sagon, X. Faurel, J. Raman Spectrosc. 32, 35160 (2001)

23. P. Colomban, I. Robert, C. Roche, G. Sagon, V. Milande, Rev. Archeom. 28, 153-167 (2004)

24. E. Radice, Analysis of Raman Spectra of Mosaic Glasses. Bachelor Thesis, Physics Degree, University of Milano Bicocca (2019)

25. P. Colomban, J. Non Cryst. Solids 323, 180187 (2003)

26. E. Basso, C. Invernizzi, M. Malagodi, M.F. La Russa, D. Bersani, P.P. Lottici, J. Raman Spectrosc. 45, 238245 (2014)

27. http://atom.kaeri.re.kr/nuchart. Accessed 9th Mar 2021

28. R.H. Brill, The Corning Museum of Glass (1970), pp. 105-128

29. S. Fiorentino et al., Archaeol. Anthropol. Sci. 11, 337-359 (2019)

30. https://www.nist.gov/laboratories/tools-instruments/instrumental-neutron-activation-analysis-inaa. Accessed 9 Mar 2021 